\title{
Chlorpyrifos-Induced Delayed Neurotoxicity with A Rare Presentation of Flaccid Quadriplegia: A Diagnostic Challenge
}

\author{
Patrícia Afonso Mendes ${ }^{1}$, Tatiana Cunha Pereira ${ }^{2}$, Rui Pina ${ }^{1}$, Rui Santos ${ }^{1}$ \\ ${ }^{1}$ Internal Medicine Department A, Centro Hospitalar e Universitário de Coimbra, Coimbra, Portugal \\ ${ }^{2}$ Oncology Service, Instituto Português de Oncologia de Coimbra Francisco Gentil, Coimbra, Portugal
}

Received: 27/09/2017

Accepted: 07/10/2017

Published: 30/10/2017

How to cite this article: Afonso Mendes P, Cunha Pereira T, Pina R, Santos R. Chlorpyrifos-induced delayed neurotoxicity with a rare presentation of flaccid quadriplegia: a diagnostic challenge. EJCRIM 2017;4: doi:10.12890/2017_000751

Conflicts of Interests: The Authors declare that there are no competing interests.

Acknowledgements: The authors would like to thank Dr Argemiro Geraldo and Joana Jesus Ribeiro from the Department of Neurology, Centro Hospitalar e Universitário de Coimbra, for invaluable help and guidance throughout the follow-up of this patient. We would also like to acknowledge Orlando Galego from the Neuroradiology Department for providing the images presented in this case report.

This article is licensed under a Commons Attribution Non-Commercial 4.0 License

\section{ABSTRACT}

Chlorpyrifos is an organophosphate compound recognized as causing acute toxicity. However, organophosphate-induced delayed polyneuropathy (OPIDP), although rare, has also been described. We describe an unusual presentation of OPIDP with flaccid quadriplegia progressing to a locked-in-like syndrome, 30 days after a 60-year-old man voluntarily ingested chlorpyrifos. In the absence of specific treatment, the patient only recovered partial motor responses and the ability to communicate. The authors present this report in order to highlight a form of OPIDP which can hinder diagnosis due to its atypia and the delay in the onset of symptoms from initial contact with the toxicant.

\section{LEARNING POINTS}

- Organophosphate-induced delayed polyneuropathy (OPIDP) is a late presentation which is often overlooked despite causing significant morbidity.

- OPIDP can present in several forms, hindering the final diagnosis and consuming resources.

\section{KEYWORDS}

Organophosphate poisoning, delayed neuropathy, quadriplegia

\section{BACKGROUND}

Organophosphates are known for their muscarinic and nicotinic toxicity occurring within minutes to hours after exposure ${ }^{[1]}$. A rare third toxic effect is organophosphate-induced delayed polyneuropathy (OPIDP), characterized by degeneration of long axons in both the central and peripheral nervous systems, and subsequent ataxia and paralysis, which generally appear 2-3 weeks or more after organophosphate exposure ${ }^{[2]}$. A common physical finding is spastic paraparesis. We report a rare case of flaccid quadriplegia with a locked-in-like syndrome, in a man who voluntarily ingested a chlorpyrifos compound. 


\section{CASE REPORT}

A 60-year-old patient was admitted to the intensive care unit with respiratory depression requiring mechanical ventilation, after voluntarily ingesting $100 \mathrm{ml}$ Nufos $480^{\circledR}$ (chlorpyrifos organophosphate). He was promptly treated with atropine perfusion and obidoxime with progressive clinical improvement. The patient was then transferred to the internal medicine ward for treatment for ventilator-associated pneumonia caused by a multi-sensitive Staphylococcus aureus. Twenty-eight days after ingestion, the patient began to experience diaphoresis, tachycardia, dyspnoea, leg pain and cramps, and fell at the bedside. Shortly thereafter, he showed flaccid paraparesis (muscle strength G2) and hyperaesthesia with rapid caudal progression, followed by urinary retention and priapism, and escalating to a locked-in-like syndrome 1 week after the onset of the neurological impairment. Despite this rapid evolution and the quadriplegia, the patient did not have significant respiratory failure or need mechanic ventilation, with normal blood gas analysis under supplementary oxygen ( $2 \mathrm{l} / \mathrm{min})$. The patient was tachypnoeic and used his eyes to answer simple questions. He also had a high temperature $\left(41^{\circ} \mathrm{C}\right.$ maximum) that only partially responded to four concomitant antipyretics and lasted for several weeks.

Serum cholinesterase remained low for 3 weeks. The serum auto-immunity study was negative for all antibodies although there was weak positivity for anti-CV2 antibodies. Cerebrospinal fluid (CSF) only showed slightly elevated glucose (73 mg/dl, normal value < $70 \mathrm{mg} / \mathrm{dl})$ with negative virology and cultures. However, cytomegalovirus (CMV) DNA was seen in serum despite normal immunoglobulins and absence of CMV DNA in CSF.

Electroencephalography demonstrated moderate slowing of electrical activity, while electromyography showed severe bilateral lumbosacral radiculopathy, with no signs of sensory or motor polyneuropathy.

Magnetic resonance imaging (MRI) exhibited confluent lesions with diffuse and bilateral involvement, which was mainly peri/para ventricular, mostly posterior but also infratentorial, with cortical-subcortical involvement more evident in the temporal and parietal lobes on the right (Fig. 1). Intra-medullary lesions with increased T2 and T2 STIR signals were also observed in C2, C3, C4, D1 and D5-D8 (Fig. 2).

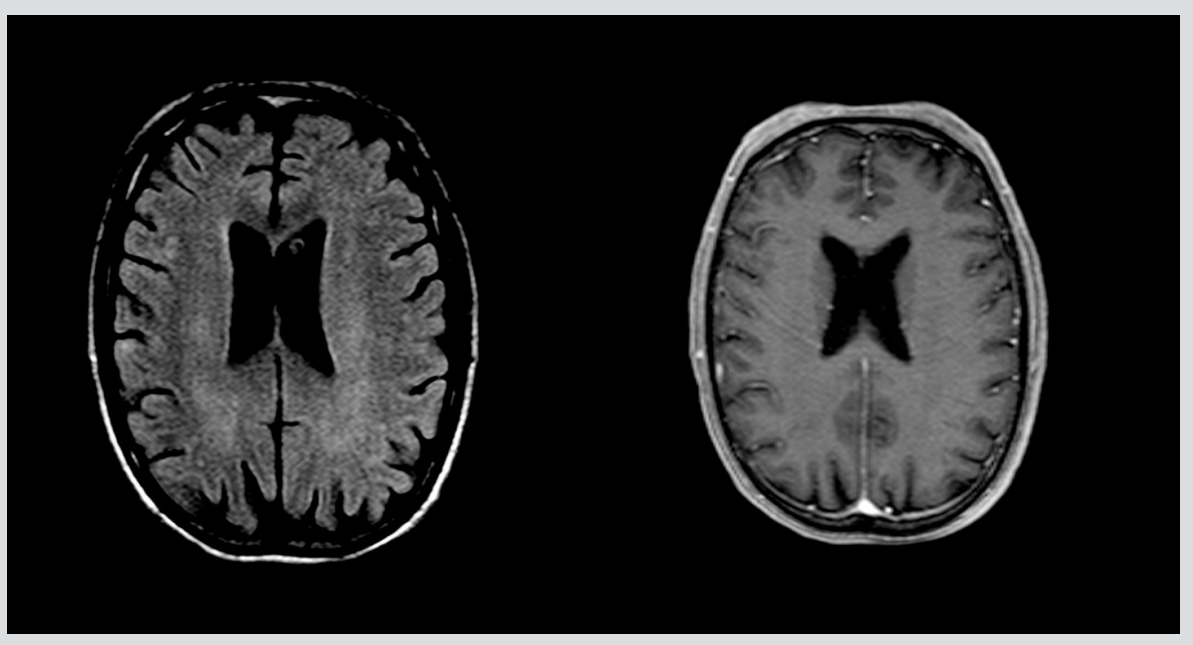

Figure 1. Confluent lesions with diffuse and bilateral involvement, mainly peri/para ventricular, mostly posterior but also infratentorial, with cortical-subcortical involvement more evident in the temporal and parietal lobes on the right injections

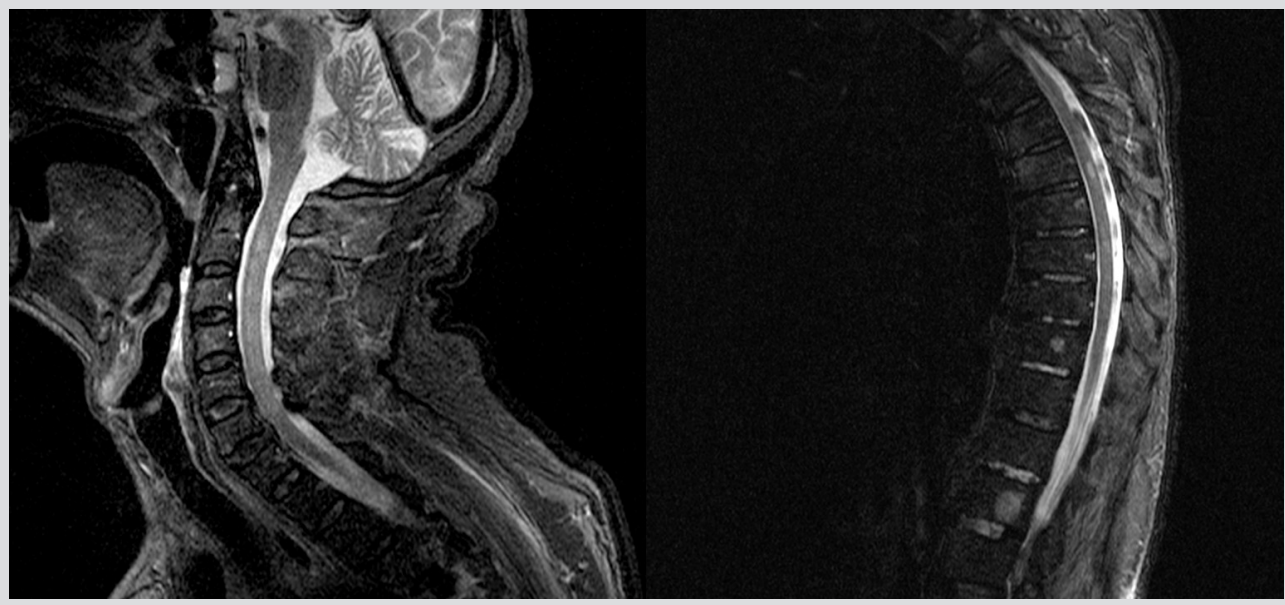

Figure 2. Intra-medullary lesions with increased T2 and T2 STIR signals were also observed in C2, C3, C4, D1 and D5-D8 
The clinical presentation and the results of investigations suggested delayed neurotoxicity due to chlorpyrifos. However, since the patient developed very high temperatures and was positive for serum CMV DNA, Guillain-Barré syndrome with a locked-in-like syndrome was also considered and treated, despite the absence of the respiratory failure expected with such neurological involvement. The possibility of an auto-immune disorder was considered but rejected due to investigation results and lack of improvement following initial treatment with three pulses of $1,000 \mathrm{mg}$ of methylprednisolone, intravenous immunoglobulins and plasmapheresis.

The patient slowly recovered partially, at discharge demonstrating G4 strength in the superior limbs but maintaining flaccid paraplegia with recovery of thermoregulatory and tactile sensitivity. One year after the onset of symptoms, the patient maintains the motor deficits, now with spasticity and hyperreflexia in both lower limbs but with normal strength in his arms and is able to communicate normally. A new MRI showed partial resolution of the medullar alterations with some lesions progressing to chronicity.

\section{DISCUSSION}

This case was a diagnostic challenge because of the atypical presentation of OPIDP, which is a rare organophosphate neurotoxicity occurring 1-5 weeks after significant contact with organophosphate compounds such as chlorpirifos ${ }^{[2,3]}$.

Its clinical presentation begins with cramp-like pain in the calves which becomes neuropathic, as well as motor signs and symptoms that begin peripherally and rapidly progress proximately and include paraesthesias, paralysis, ataxia and depression of deep tendon reflexes. Frequently these patients show paraparesis but in severe cases may present with spastic quadriparesis ${ }^{[2,4]}$. Atypical presentations have also been described ${ }^{[1,5]}$.

Although our patient's locked-in-like syndrome could have been secondary to CMV infection as serum CMV DNA was present, the absence of CMV DNA in CSF, and the lack of serum IgM antibodies and of response to timely treatment, makes this diagnosis unlikely.

Atropine, obidoxime and corticosteroids might have some effect on the progression of OPIDP symptoms, but no specific treatment, with reversal of the clinical course, is known ${ }^{[2,4]}$.

We believe that this patient's clinical course and investigation results were compatible with a rare OPIDP presentation with prolonged flaccid quadriplegia and a locked-in-like syndrome, evolving to chronic paraplegia. This case report should alert clinicians to the different forms of OPIDP and help prevent delayed diagnosis.

\section{REFERENCES}

1. Lotti M, Moretto A. Organophosphate-induced delayed polyneuropathy. Toxicol Rev 2005;24:37-49.

2. Vale A, Lotti M. Organophosphorus and carbamate insecticide poisoning. Handb Clin Neurol 2015;131:149-168.

3. Thivakaran T, Gamage R, Gunarathne KS, Gooneratne IK. Chlorpyrifos-induced delayed myelopathy and pure motor neuropathy: a case report. Neurologist 2012;18:226-228.

4. Jokanovic M, Stukalov PV, Kosanovic M. Organophosphate induced delayed polyneuropathy. Current Drug Targets CNS Neurol Disord 2002;1:593-602.

5. Merwin SJ, Obis T, Nunez Y, Re DB. Organophosphate neurotoxicity to the voluntary motor system on the trail of environment-caused amyotrophic lateral sclerosis: the known, the misknown, and the unknown. Arch Toxicol 2017;91(8):1-14. 